\title{
Successful treatment of COVID-19 induced neutrophilic myositis with intravenous immunoglobulin and corticosteroids: a case report
}

\author{
S. Assar, M. Pournazari, P. Soufivand, D. Mohamadzadeh \\ Rheumatology Department, Clinical Research Development Center, Imam Reza Hospital, \\ Kermanshah University of Medical Sciences, Kermanshah, Iran
}

\begin{abstract}
SUMMARY
Neutrophilic myositis (NM) is an inflammatory disorder predominantly characterized by neutrophilic infiltration in the muscles, which is suggested to be an extracutaneous manifestation of neutrophilic dermatosis (ND). $\mathrm{NM}$ is a rare disorder which has been occasionally reported in association with hematologic and inflammatory disorders. This case report describes a 45-year-old woman who presented with gradual muscle weakness developed after coronavirus disease 2019 (COVID-19) infection. Electromyography and nerve conduction velocity findings were compatible with inflammatory myopathy and muscle biopsy revealed neutrophilic infiltration. She was successfully treated with intravenous immunoglobulin, prednisolone $1 \mathrm{mg} / \mathrm{kg} / \mathrm{day}$ and azathioprine $150 \mathrm{mg} /$ day. In conclusion, the COVID-19 infection itself and the drugs used to treat it can cause a number of muscle disorders. Awareness of muscular involvement in COVID-19 infected patients is important for early diagnosis and appropriate treatment.
\end{abstract}

Key words: COVID-19, neutrophilic myositis, SARS-CoV-2, intravenous immunoglobulins.

\section{INTRODUCTION}

N eutrophilic myositis (NM) is a very rare disorder histologically characterized by dense neutrophil infiltration in skeletal muscles (1-7). The exact etiology and pathogenesis of this disease is unclear, but it is believed to be within the spectrum of neutrophilic disease such as acute febrile neutrophilic dermatosis (AFND), also known as Sweet syndrome (1). Similar to AFND, NM has been associated with underlying hematologic and inflammatory diseases, such as acute myeloid leukemia (AML) (1-4), ulcerative colitis (UC) (5), celiac disease (6), myelodysplastic syndrome (7) and systemic lupus erythematous (8). In our patient, NM developed following coronavirus disease 2019 (COVID-19) infection. It is still unclear whether the novel coronavirus, severe acute respiratory syndrome coronavirus 2 (SARS-CoV-2), was actually an etiologic factor or not.

\section{CASE REPORT}

A 45-year-old woman presented with muscle weakness in both thighs and arms. The symptoms gradually progressed within 2 months, and she became unable to perform routine daily activities. She denied pain or paresthesia in limbs. In March 2021, she was admitted to the rheumatology department at Kermanshah University, Iran for work-up. Her past pharmacological history included 4 years of thyroxin supplementation for hypothyroidism, propranolol and ergotamine for migraine attacks, and several years of duloxetine and chlordiazepoxide for obsessive-compulsive disorder. She was infected with COVID-19 about 4 months prior to the present hospitalization. Her symptoms at that time included: fever, fatigue, cough and dyspnea. Due to severe pulmonary involvement and very low blood oxygen saturation she was admitted to the Intensive Care Unit and supported with me- 
chanical ventilation. Polymerase chain reaction test for COVID-19 was positive. Remdesivir, high doses of corticosteroids and colchicine were administered, and plasmapheresis was performed. Some early studies demonstrated the efficacy of colchicine and plasmapheresis in the treatment of COVID-19 infection $(9,10)$. She received 10,000 units of subcutaneous unfractionated heparin in two separate doses as prophylaxis of deep venous thrombosis. Most of her symptoms improved, but she continued to require supplemental oxygen through a facial mask. After six weeks, she was discharged with supplemental oxygen, $25 \mathrm{mg}$ prednisolone/ day and colchicine. Two weeks later, she started to experience muscle weakness. For suspected drug-induced myopathy corticosteroid was tapered and colchicine was discontinued. However, muscle weakness persisted and she was referred to the rheumatology clinic for further workup.

On the day of admission, the vital signs were stable, and she was not febrile. She continued to receive oxygen supplementation. During the examination of her respiratory system, diffuse crackles were reported. On neurological examination, sensation was intact and deep tendon reflexes were normal and symmetrical. Muscle strength was evaluated as 4/5 in both arms on Medical Research Council (MRC) scale. Moderate to severe weakness in the lower limbs with proximal dominant distribution was detected (MRC 3/5 in the right thigh and $2 / 5$ in the left). Gowers' sign was positive. No tenderness or swelling were reported at muscle palpation. The rest of the examination was unremarkable.

The laboratory findings on the admission day are reported in Table I. Additional laboratory workup showed thyroid stimulating hormone $3.8 \mathrm{uIU} / \mathrm{mL}$ (normal: 0.27-4.2 uIU/mL), T4 $7.92 \mathrm{ug} / \mathrm{dL}$ (normal: 5.13-14.06 ug/dL). PTH was $20.6 \mathrm{pg} / \mathrm{mL}$ (normal: 15-65 pg/mL). Immunological blood tests revealed normal results for antinuclear antibody, anti-ds DNA, antiphospholipid, anti-Ro, anti-La, ANCA, and anti Jo1 antibodies.

Electromyography and nerve conduction velocity studies (EMG/NCV) were compatible with inflammatory myopathy. There was no evidence of neuropathy and radiculopathy. Chest computerized tomography (CT) scan revealed peripheral and multi-lobar fibrotic areas in the lingula, right middle lobe and upper zones which were consistent with fibrotic changes due to previous COVID-19 infection. Echocardiography was normal with an ejection fraction of $55 \%$ and normal pulmonary artery pressure. Muscle biopsy specimen from the quadriceps revealed neutrophilic infiltration consistent with myositis. There was no evidence of vacuoles, granulomas, eosinophilic infiltrations, or vasculitis (Figure 1). She was diagnosed with neutrophilic myositis. Intravenous immunoglobulin (IVIG, $2 \mathrm{~g} / \mathrm{kg}$ in four divided doses) along with prednisolone $1 \mathrm{mg} / \mathrm{kg} /$ day were started with gradual tapering. An impressive

Table I - Laboratory findings of the patient on admission.

\begin{tabular}{|l|c|c|}
\hline Parameters (units) & Result & Normal values \\
\hline $\mathrm{Hb}$ (g/dL) & 12.2 & $12-14$ \\
\hline WBC (K/uL) & 18,2 & \\
PMN & $78 \%$ & $4-10$ \\
Lymph & $20 \%$ & \\
\hline Platelet (K/uL) & 538 & $150-400$ \\
\hline PTT (seconds) & 25 & $24-35$ \\
\hline INR & 1 & 1 \\
\hline Serum creatinine & 0.7 & $0.6-1.4$ \\
(mg/dL) & 9.5 & $8.3-11$ \\
\hline Ca (mg/dL) & 4.2 & $2.5-5$ \\
\hline P (mg/dL) & 2.5 & $1.8-2.6$ \\
\hline Mg (mg/dL) & $2+$ & Negative-4+ \\
\hline CRP (qualitative) & 87 & $<25$ \\
\hline ESR (mm/1sthr) & 445 & $225-500$ \\
\hline LDH (IU/mL) & 162 & $5-200$ \\
\hline CPK (IU/L) & 4.8 & Up to 7.6 \\
\hline Aldolase (IU/L) & 73 & $5-40$ \\
\hline AST (IU/L) & 69 & $5-40$ \\
\hline ALT (IU/L) & & \\
\hline & & \\
\hline
\end{tabular}

$\mathrm{Hb}$, hemoglobin; WBC, white blood cells; PTT, partial thromboplastin time; INR, International normalized Ratio; ESR, erythrocyte sedimentation rate; CRP, C-reactive protein; $\mathrm{LDH}$, lactate dehydrogenase; CPK, creatine phosphokinase; AST, aspartate transaminase; ALT, alanine transaminase; $\mathrm{Ca}$, calcium; Mg, magnesium; P, phosphorus. 

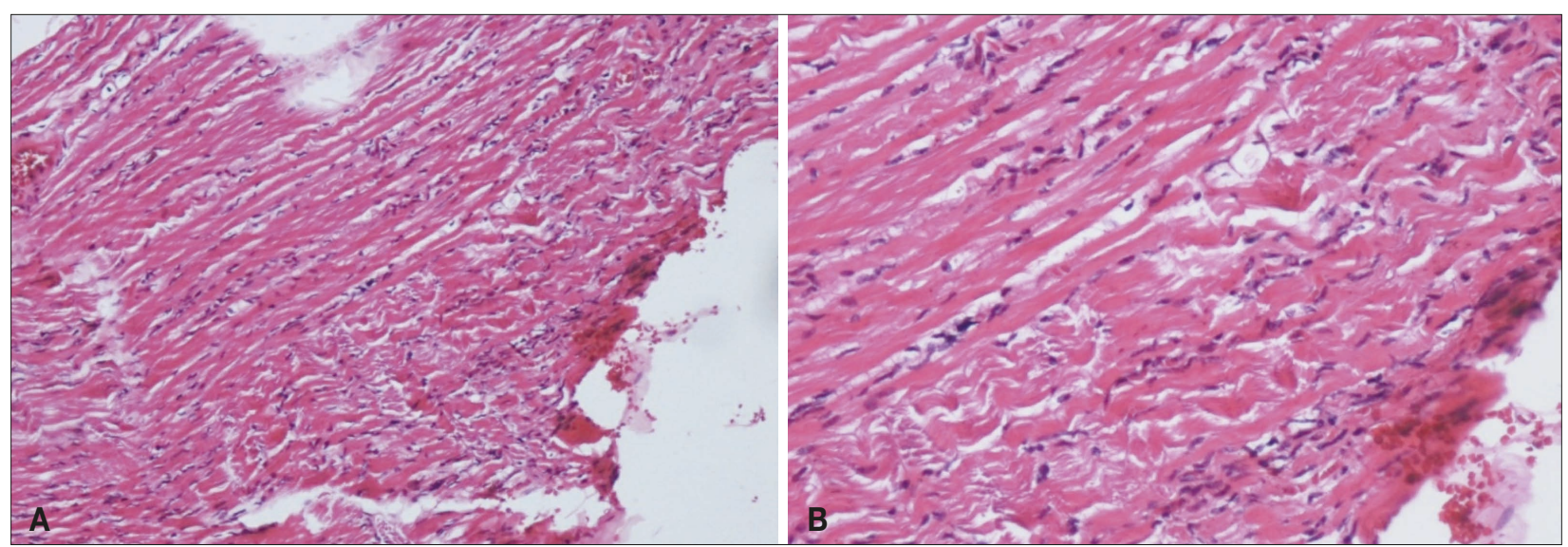

Figure 1 - A) Muscle biopsy specimen from the quadriceps showing neutrophilic infiltration (hematoxylin-eosin stain, $\times 100)$ and B) at higher magnification (hematoxylin-eosin stain, $\times 200$ ).

improvement in muscle strength occurred within 14 days. Similar significant responses were observed in previous reports. She was discharged with prednisolone $25 \mathrm{mg}$ daily and azathioprine $150 \mathrm{mg}$ daily. She was followed up in the rheumatology clinic after discharge and remains on a maintenance therapy with oral prednisolone $5 \mathrm{mg}$ daily and azathioprine.

\section{DISCUSSION AND CONCLUSIONS}

$\mathrm{NM}$ is a rare disease characterized by a pathological infiltration of neutrophils in muscle tissues. The etiology and pathogenesis of this disease is unclear, but it is suspected to be within the spectrum of neutrophilic disease such as AFND (1) As far as we know, only 8 cases (1-8) have been reported so far. All the reported cases were associated with several hematologic and inflammatory disorders such as acute myeloid leukemia, myelodysplastic syndrome, celiac disease, ulcerative colitis or Crohn's disease and systemic lupus erythematous. However, in the last one, the authors were unable to confirm NM histologically in the muscles of the lupus patient, but neutrophilic infiltration was observed in the dermis and subcutaneous tissue of a skin biopsy. As far as we know, no previous article has ever reported a case of NM in association with viral or bacterial infections. In our patient, other specific disorders such as electrolyte imbalances, hypothyroidism, colchicine-induced myopathy, polymyositis (PM) and dermatomyositis (DM) and hematologic disorders were excluded based on laboratory, clinical and pathological findings. Colchicine myopathy is characterized by vacuolar changes and the muscle biopsy often contains some basophilic material $(11,12)$ and PM or DM are associated with predominantly lymphocytic infiltration in muscle biopsy specimens. On the contrary, in this case, the infiltration was neutrophilic and there was no vacuole in the muscle biopsy specimen.

Unlike previously reported cases, our patient had no muscle tenderness or swelling, probably due to the use of anti-inflammatory drugs such as prednisolone and colchicine before the onset of disease. Accordingly, also muscle enzyme levels were within the normal limits in our case, probably for the same reason. In line with our observation, Mosnier et al. reported a 66-year-old man diagnosed with neutrophilic myositis who had normal levels of creatine phosphokinase (7).

There are only few case reports of COVID-19 associated myositis (13-15) and scanty histological studies revealed an immune-mediated mechanism or vasculitis with predominantly lymphocytic infiltrations of the muscle $(14,16,17)$. Although ACE2 (angiotensin-converting enzyme 2), the SARS-CoV-2 receptor, is suggested to be expressed in the skeletal muscles, there 
is still no evidence that the COVID-19 virus directly invades muscles (18).

The respiratory system could be involved in AFND, although infrequently, being a possible a differential diagnosis for our patient. She had a history of respiratory manifestations that progressed to respiratory failure needing mechanical ventilation. Corticosteroid-responsive, culture-negative infiltrates and pulmonary tissue with neutrophilic inflammation are signs of pulmonary involvement of AFND. We did not perform a lung tissue biopsy from the patient, but the CT scan showed fibrotic areas which are not typical of lung involvement in AFND and made this diagnosis less likely (19).

To the best of our knowledge, our case is the first report of NM developed in a patient previously infected with COVID-19. This may indicate that the COVID-19 infection can potentially lead to a wide spectrum of muscle disorders.

In conclusion COVID-19 infection, as well as the drugs used to treat it, can cause a variety of muscle disorders that must be identified. Further studies are needed to clarify the muscular manifestations associated with COVID-19 infection.

\section{Informed Consent}

An informed written consent was obtained from the patient to publish her clinical data and the images.

\section{Conflict of interests}

The authors declare that they have no conflicts of interests.

\section{Funding}

This research did not receive any specific grant from funding agencies in the public, commercial or not-for-profit sectors.

\section{REFERENCES}

1. Marie I, Levesque H, Joly P, et al. Neutrophilic myositis as an extracutaneous manifestation of neutrophilic dermatosis. J Am Acad Dermatol. 2001; 44: 137-9.

2. Kim MK, Park JW, Park SH, et al. Neutrophilic myositis without cutaneous involvement as the first manifestation of acute myeloid leukemia. Korean J Intern Med. 200; 20: 346-8.

3. Christ E, Linka A, Jacky E, et al. Sweet's syndrome involoving the musculoskeletal system during treatment of promyelocytic leukemia with all-trans retinoic acid. Leukemia. 1996; 10: 731-4.

4. Melinkeri SR, Gupta RK, Dabadghao S. A Sweet-like syndrome manifesting as gingival hyperplasia and myositis without cutaneous involvement. Ann Hematol. 2002; 81: 397-8.

5. Qureshi JA, Staugaitis SM, Calabrese LH. Neutrophilic myositis: an extra-intestinal manifestation of ulcerative colitis. J Clin Rheumatol. 2002; 8: 85-8.

6. Alawneh K, Ashley C, Carlson JA. Neutrophilic myositis as a manifestation of celiac disease: a case report. Clin Rheumatol. 2008; 27: 11-3.

7. Mosnier E, Rousset P, Hugol D, et al. Neutrophilic myositis sine dermatitis in a neutropenic patient with myelodysplastic syndrome. Ann Hematol. 2013; 92: 551-2.

8. Matsui Y, Makino T, Torai R, Shimizu T. Neutrophilic myositis developing in a case of systemic lupus erythematosus. Eur J Dermatol. 2020; 30: 432-433.

9. Parra-Medina R, Sarmiento-Monroy JC, Rojas-Villarraga A, et al. Colchicine as a possible therapeutic option in COVID-19 infection. Clin Rheumatol. 2020; 39: 2485-6.

10. Balagholi S, Dabbaghi R, Eshghi P, et al. Potential of therapeutic plasmapheresis in treatment of COVID-19 patients: Immunopathogenesis and coagulopathy. Transfus Apher Sci. 2020; 59: 102993.

11. Kuncl RW, Duncan G, Watson D, et al. Colchicine myopathy and neuropathy. N Engl J Med. 1987; 316: 1562-8.

12. Riggs JE, Schochet SS Jr, Gutmann L, et al. Chronic human colchicine neuropathy and myopathy. Arch Neurol. 1986; 43: 521-3.

13. Uslu S. Myositis due to COVID-19. Postgrad Med J. 2021; 97: 399.

14. Zhang H, Charmchi Z, Seidman RJ, et al. COVID-19-associated myositis with severe proximal and bulbar weakness. Muscle Nerve. 2020; 62: 57-60.

15. Beydon M, Chevalier K, Al Tabaa O, et al. Myositis as a manifestation of SARS-CoV-2. Ann Rheum Dis. 2020; 23: 2020-217573.

16. Leung TW, Wong KS, Hui AC, et al. Myopathic changes associated with severe acute respiratory syndrome: a postmortem case series. Arch Neurol. 2005; 62: 1113-7.

17. Manzano GS, Woods JK, Amato AA. Covid19-associated myopathy caused by type I interferonopathy. N Engl J Med. 2020; 383: 23892390.

18. Dalakas MC. Inflammatory myopathies: update on diagnosis, pathogenesis and therapies, and COVID-19-related implications. Acta Myol. 2020; 39: 289-301.

19. Cohen PR. Sweet's syndrome - a comprehensive review of an acute febrile neutrophilic dermatosis. Orphanet J Rare Dis. 2007; 2: 34. 